\title{
Prolonged Fecal Shedding of SARS-CoV-2 in Asymptomatic Children with Inborn Errors of Immunity
}

\author{
Madhu Chhanda Mohanty ${ }^{1}$ [ $\cdot$ Prasad D. Taur $^{2} \cdot$ Unnati Prashant Sawant ${ }^{1} \cdot$ Reetika Malik Yadav $^{3} \cdot$ Varsha Potdar $^{4}$
}

Received: 4 August 2021 / Accepted: 31 August 2021 / Published online: 16 September 2021

(c) The Author(s), under exclusive licence to Springer Science+Business Media, LLC, part of Springer Nature 2021

To the Editor,

SARS-CoV-2 (severe acute respiratory syndrome coronavirus 2) infection responsible for COVID-19 pandemic has affected more than 100 million cases worldwide. Although fewer number of cases have been reported in children, they remain undetected as they are asymptomatic or mildly symptomatic, creating a threat of unknown SARS-CoV-2 transmission to the community [1]. One of the characteristics of pediatric SARS-CoV-2 infection is the persistent fecal viral shedding despite negative nasopharyngeal swab testing, thereby raising the possibility of feco-oral transmission [2]. Pre-existing co-morbidities and an inborn defect in immunity may contribute to a more severe course of COVID-19 in children. Inborn errors of immunity (IEI) are group of more than 400 inherited disorders and patients with some IEI are at an increased risk of developing severe COVID-19 [3].

Prolonged excretion of polioviruses in children suffering from IEI immunized with oral poliovirus vaccine (OPV) or exposed to environmental poliovirus leading to viral mutations has been reported by several studies [4]. In a similar context, these children may also act as a reservoir of SARS$\mathrm{CoV}-2$ infection. Reports of prolonged viral carrier state in immunocompromised patients have underlined the risk for within-host variant generation as seen in the B.1.1.7 variant [5]. Therefore, studies on the susceptibility of individual IEI

Madhu Chhanda Mohanty

madhuerc@gmail.com

1 ICMR-National Institute of Virology, Mumbai Unit, Indian Council of Medical Research, Haffkine Institute Campus, Acharya Donde Marg, Parel, Mumbai 400012, India

2 Bai Jerbai Wadia Hospital for Children, Mumbai, Maharashtra, India

3 ICMR-National Institute of Immunohaematology, Mumbai, Maharashtra, India

4 ICMR-National Institute of Virology, National Influenza Center (NIC), Pune, India patients to COVID-19 are relevant for these patients as well as for the community.

We report our findings from a study conducted in a small cohort of pediatric patients with IEI to investigate the fecal shedding of SARS-CoV-2 at a tertiary health care center in Mumbai. The stool samples from diagnosed IEI patients were tested retrospectively for SARS-CoV-2 after due approval from ethics committee of BJWHC, Mumbai, and ICMR-NIV, Pune. A total number of 68 stool samples from 34 children (collected from March to October 2020) diagnosed with 17 different IEIs were tested for SARS-CoV-2 infection (Table S1).

The clinical, immunological, and molecular findings; treatment details; and symptoms at time of stool sample collection along with the results of stool and nasopharyngeal swab testing of the four patients tested positive are shown in Table 1. Patient 1 (P1) diagnosed as hyper IgM syndrome was observed to shed the virus for about 99 days from the day first tested positive. The content of nucleic acid decreased with increase in months and stopped after 99 days. The $\mathrm{Ct}$ values ranged from 26.97 in Orf1ab gene on day 1 to 34.19 on day 99 in the 4 samples consecutively collected with an interval of approximately a month. Patient 2 (P2) diagnosed with Wiskott-Aldrich syndrome shed the virus for nearly 53 days. The $\mathrm{Ct}$ value in Orfl ab target gene on day 1 was 22.98 , which increased on day 21 , but a significant decrease was observed on day 53. Patient 3 (P3) and patient $4(\mathrm{P} 4)$ showed SARS-CoV-2 virus shedding on day 1 and later tested negative in the consecutive samples (Table 1, Fig. 1).

Further extensive analysis of SARS-CoV-2 positive stool samples and respective nasal swab (NS)/throat swab (TS) samples at National Influenza Center (NIC), ICMR-NIV, Pune, confirmed SARS-CoV-2 detection in stool samples; in addition, another aliquot of the NS/TS sample of P1 was also found positive for SARS-CoV-2. The viral load for the follow-up stool samples is mentioned in Table 2. The NGS data following alignment and mapping of SARS-CoV-2 


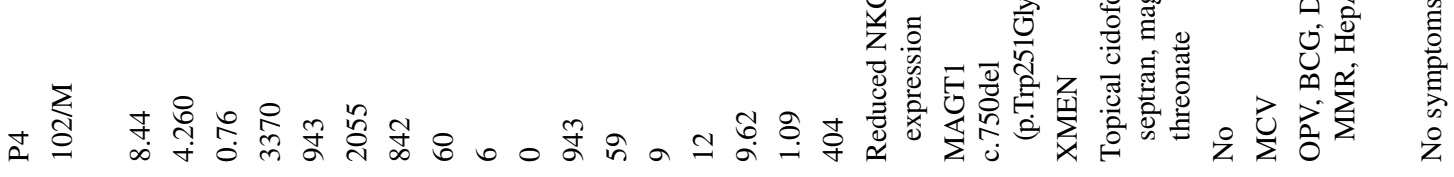

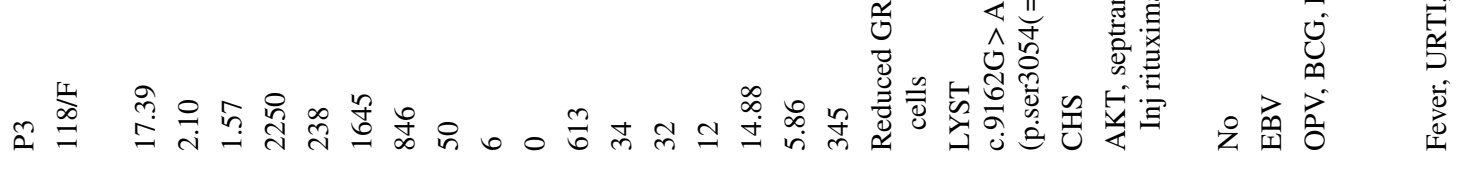

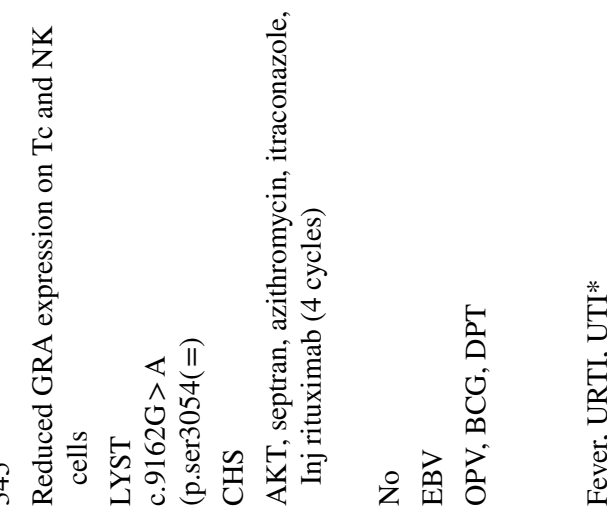

毫

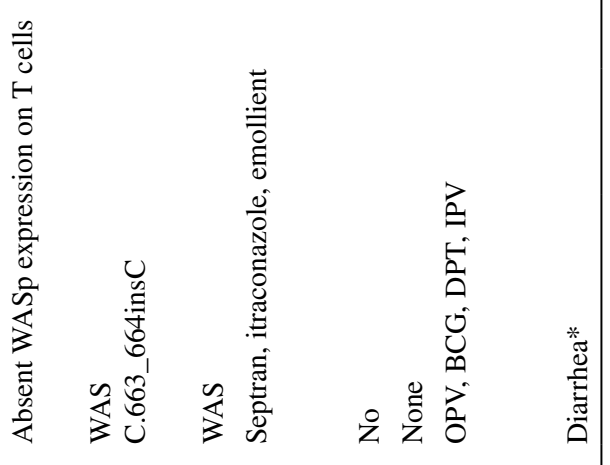

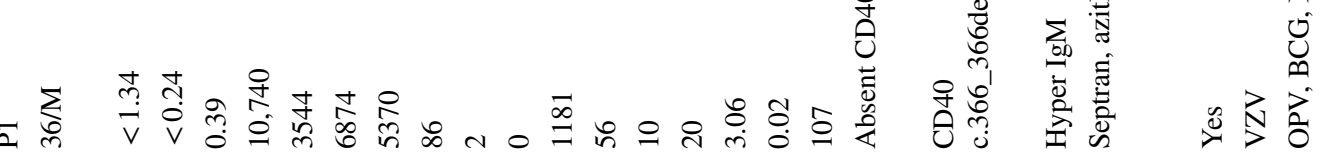
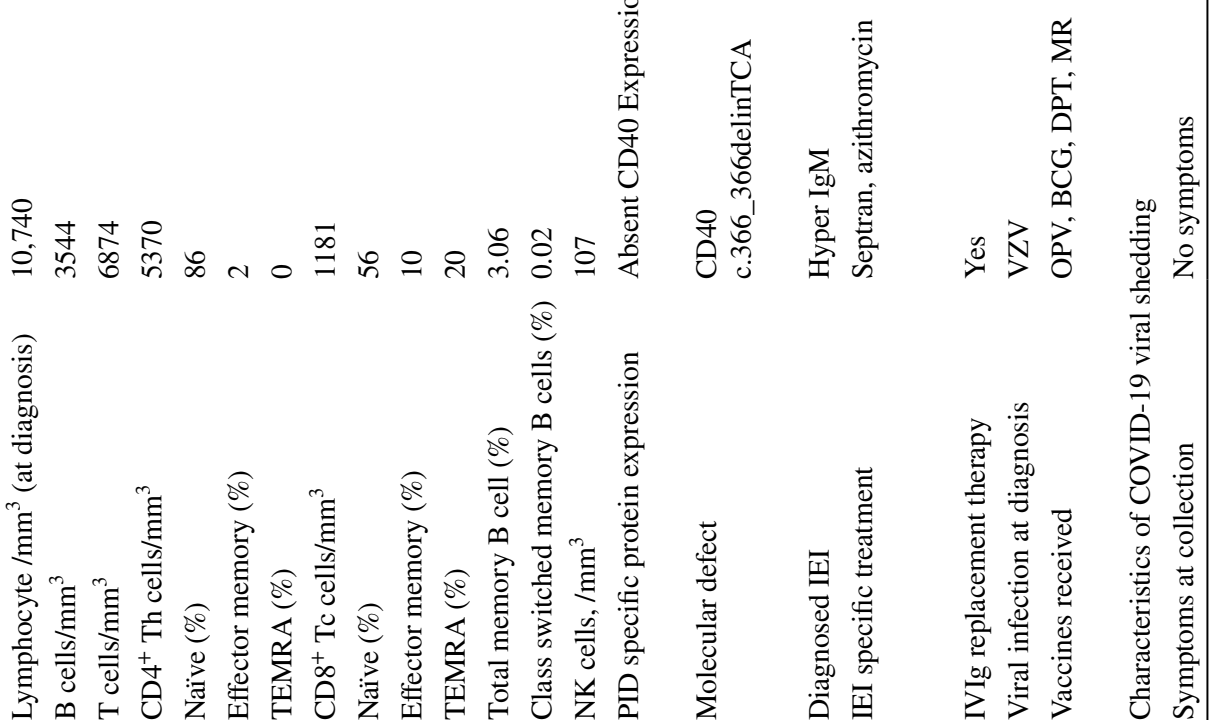


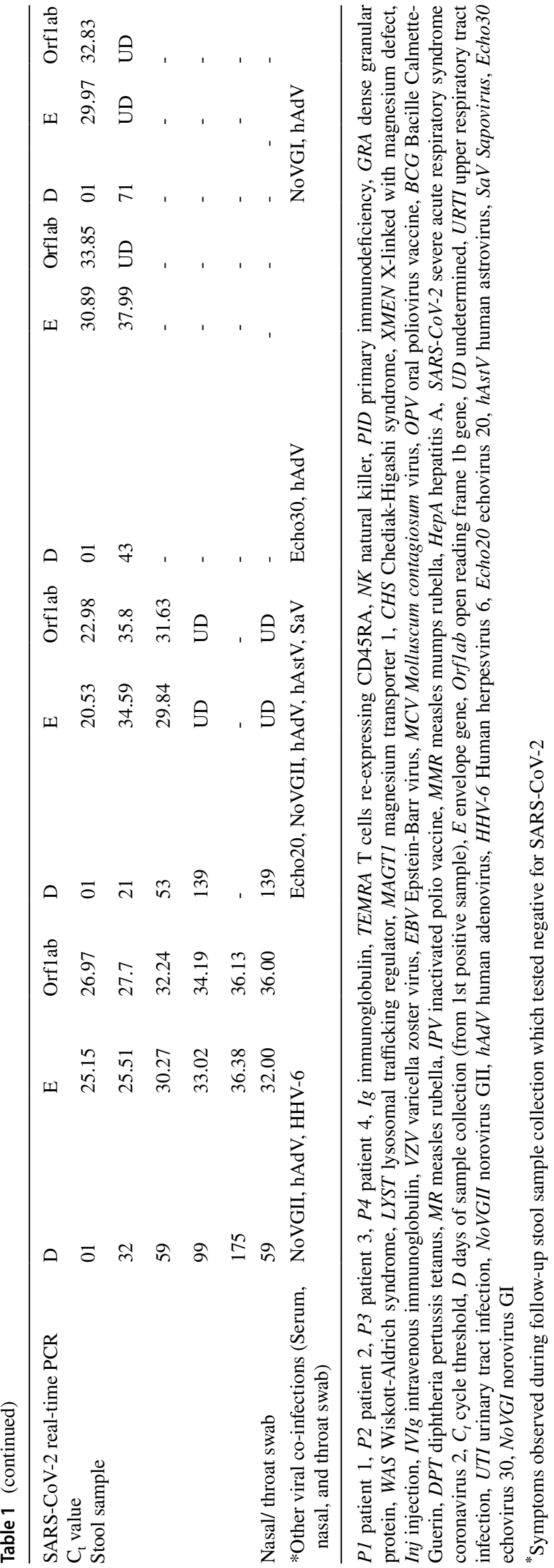

were compared to the Wuhan-Hu-1 (MN90847.3) SARSCoV-2 reference genome and mutational variations were identified in follow-up stool samples. Collectively, there were thirty-eight different mutations detected among follow-up stool samples (Table S2), and the lineage and clades were listed in Table 2. Phylogenetic analysis of the sequential samples of three patients possessing GR clade is shown in Fig. 1c.

We report prolonged excretion of SARS-CoV-2 in two pediatric patients with PAD and CID who were never symptomatic for COVID-19 and had no history of contact with known COVID-19 patient. NS and TS collected (after 1st follow-up stool sample tested positive) were tested positive for P1. The persistent shedding of SARS$\mathrm{CoV}-2$ in stool of infected immunocompetent children continually reported by new studies [2] is in accordance with our findings, showing that SARS-CoV-2 may be present in the gastrointestinal tract for a longer duration than viral presence in the respiratory system. Vero CCL81 cells to test the viability of SARS-CoV-2 detected in frozen stool samples could not detect live virus.

Fecal SARS-CoV-2 RNA detection in immunocompetent children with a viral excretion time over 46 days in some patients has been reported in a study [2]. Our findings demonstrated up to 100 days of fecal viral detection in one of the IEI patients. We would like to highlight that the exact period of SARS-CoV-2 excretion in these children may be much longer than noted by us, as these children were never tested for COVID19 prior to enrolment in the study. COVID-19 specific clinical symptoms were observed during follow-up stool sample collection in P2 and P3; however, these stool samples were found to be negative for SARS-CoV-2. Moreover, no clinical symptoms were recorded in the patients while they were positive for SARS-CoV-2 excretion.

Next-generation sequencing identified the SARS-CoV-2 whole-genome sequence of notable interest, in four sequential samples of P1. In the paired TS/NS and stool sample of P1, TS/NS sample had the least mutations and defined as $\mathrm{G}$ clade, whereas subsequent stool samples had major changes across the genome and defined as GR clade. The data indicated marked within-host genomic evolution of SARS-CoV-2, throughout the course of infection. Overall, the results showed the quasispecies' profile of SARS$\mathrm{CoV}-2$ providing insights into its genetic dynamics during the course of infection, within different sample types. The stool samples had more missense mutation than nasopharyngeal swab.

It was difficult to explain the persistent Noro and adenovirus infection in the two prolonged SARS-CoV-2 excreters in our study, as it could be IEI specific or COVID-19-related co-infection. 


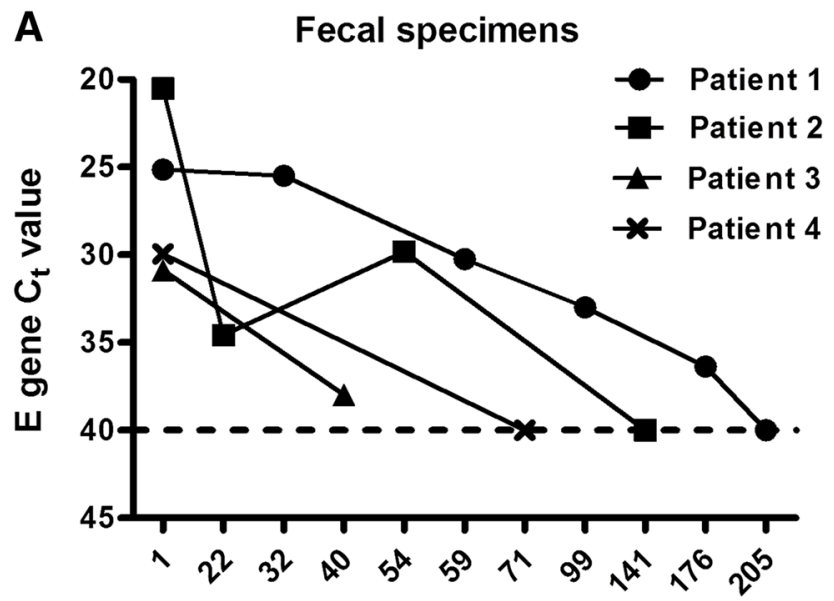

Sample collection days

\section{C}

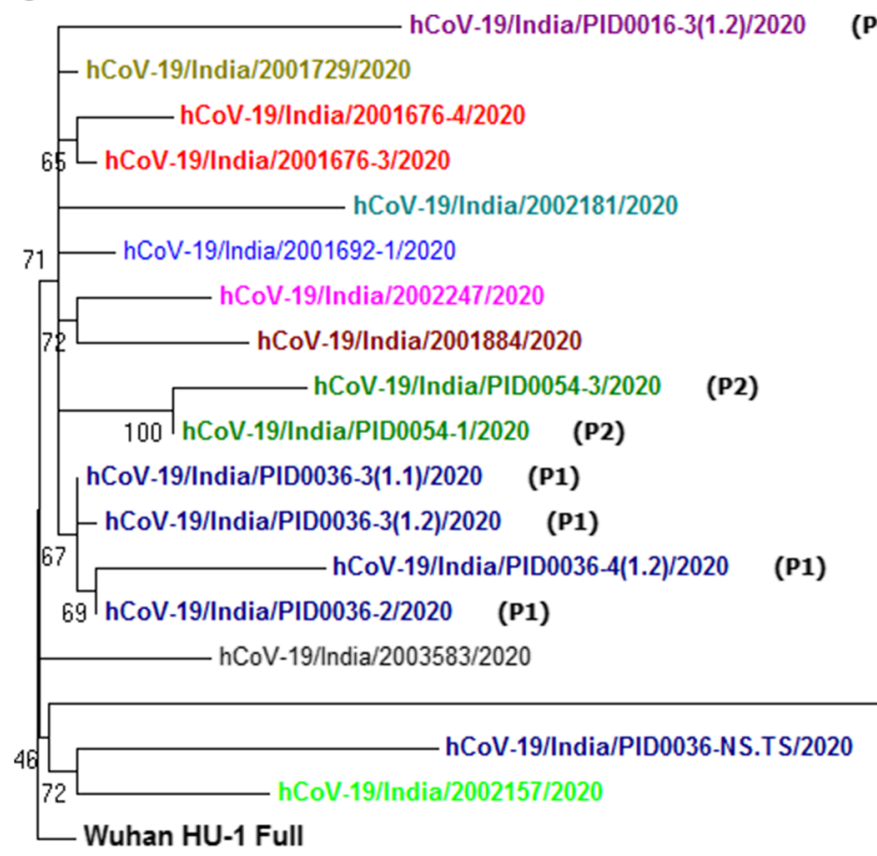

0.0005

Fig. 1 Viral load of SARS-CoV-2 detected in the children with inborn errors of immunity. Consecutive stool specimens of patients were tested using real-time RT-PCR. $Y$-axis represents $\mathrm{Ct}$ values and $X$-axis represents the intervals of sample collection (days). Ct values are marked with black solid symbols. a Distribution of $C_{t}$ values for

There are several limitations in our study. Since the children were asymptomatic and there was no history of COVID-19 in the family, the parents were hesitant to perform the tests for other COVID-19-related parameters

\section{B Fecal specimens}

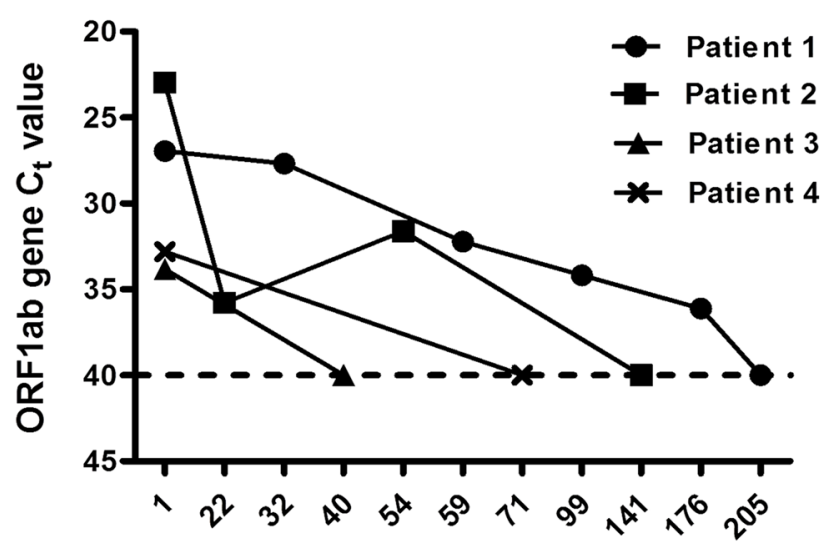

Sample collection days
(P4)

GR

hCoV-19/India/PID0036-5/2020

(P1)

(P1)

the E gene (screening gene) obtained from real-time RT-PCR of stool specimens of 4 patients. b Distribution of $C_{t}$ values for the ORF1b gene (confirmatory gene) obtained from real-time RT-PCR of stool specimens of 4 patients. $\mathbf{c}$ Phylogenetic analysis of the fecal samples for the patients excreting SARS-CoV-2

to correlate with the viral excretion. Timely collection of stool/throat/nasal swab differed due to lockdown and vulnerability of the patients towards COVID-19. The methodology of stool sample collection, storage, and 
Table 2 Next-generation sequencing analysis of SARS-CoV-2 detected in stool samples of IEI patients

\begin{tabular}{|c|c|c|c|c|c|c|c|c|c|}
\hline Patient ID & Specimen type & $\begin{array}{l}\text { Sample col- } \\
\text { lection day } \\
\text { (d) }\end{array}$ & Viral load & $\begin{array}{l}\text { Genome coverage } \\
\text { (target base coverage } \\
\text { at } 20 \times \text { ) }\end{array}$ & Mapped reads & Mean depth & $\begin{array}{l}\text { No. of } \\
\text { variants }\end{array}$ & Clade & Lineage \\
\hline \multirow[t]{5}{*}{ P1 } & Stool & d01 & 5.01E5 & $95.63 \%$ & $4,64,271$ & 2,873 & 6 & Other & B.1.1.32 \\
\hline & Stool & $\mathrm{d} 32$ & $6.3 \mathrm{E} 4$ & $97.86 \%$ & $1,16,241$ & 690.8 & 11 & GR & B.1.1.281 \\
\hline & Stool & d59 & $4.8 \mathrm{E} 3$ & $76.68 \%$ & 18,346 & 49.96 & 12 & GR & B.1.1.212 \\
\hline & Throat/nasal swabs & d59 & 190 & $84.25 \%$ & 12,877 & 52.31 & 5 & G & B.1.210 \\
\hline & Stool & d99 & $1.3 \mathrm{E} 4$ & $72.22 \%$ & $2,21,606$ & 36.46 & 6 & G & B. 1 \\
\hline \multirow[t]{2}{*}{$\mathrm{P} 2$} & Stool & d01 & 3.17E6 & $96.00 \%$ & 67,890 & 281.2 & 16 & GR & B.1.1.281 \\
\hline & Stool & $\mathrm{d} 53$ & $2.06 \mathrm{E} 4$ & $91.70 \%$ & $1,25,844$ & 672.6 & 18 & G & B.1 \\
\hline P4 & Stool & $\mathrm{d} 01$ & $4.8 \mathrm{E} 3$ & $50.53 \%$ & 6,008 & 24.2 & 2 & G & B.1.375 \\
\hline
\end{tabular}

*NGS was not performed for 2nd positive sample of P2 (i.e., day22) and 1st positive sample of P3, due to lower viral load

transportation restricted our ability to generate evidence of replication-competent virus in fecal samples.

This was the first report of prolonged detection of SARS-CoV-2 in GI tract of patients with IEI for a period of more than 99 days, highlighting the longer viral survival in the GI tract of IEI patient than anticipated. Prolonged fecal shedding of SARS-CoV-2 observed in our study highlights the potential risk of within host variant generation and feco-oral transmission in asymptomatic pediatric patients with IEI [5]. Since diarrhea is a frequent symptom in patients with IEI, there is a need for a rapid and effective modification of the screening and diagnostic algorithms of COVID-19 for these patients. These children, irrespective of the presence of symptoms of COVID-19, should be tested intermittently for SARS-CoV-2 in nasal, throat, and stool samples. A large cohort study on prolonged SARS-CoV-2 excretion in pediatric patients with IEI and related immunological parameters, viability testing, and genomic sequencing of the excreted viral particles is crucial to understand the transmission dynamics.

Supplementary Information The online version contains supplementary material available at https://doi.org/10.1007/s10875-021-01132-1.

Acknowledgements We thank the Director, ICMR-National Institute of Virology and Officer in Charge, ICMR-National Institute of Virology, Mumbai Unit, for the kind support. The authors thank Dr. Mukesh Desai, Head Dept. of Immunology, Bai Jerbai Wadia Hospital for Children and Dr. Manisha Madkaikar, Director, ICMR-National Institute of Immunohematology, for their intellectual input and critical analysis. We also thank Ms. Mevis Fernandes, Mr. Swapnil Varose, Dr. Maya Gupta, Dr. Vijaya Gowri, Ms. Ujjaini Saha, Ms. Veena Vipat, and Mr. Chandrakant Sawant for their technical support.

Author Contribution MCM conceived and designed the study; PDT and RMY participated in patient care, clinical investigation, and clinical data collection; UPS performed the experiments and analyzed the data; VP performed NGS and viral load experiments and analyzed the data; MCM, PDT, UPS, and VP interpreted the data; MCM and UPS wrote the first draft of the manuscript; MCM, RMY,
PDT, and VP critically revised the manuscript for important intellectual content. All authors drafted the manuscript for important intellectual content, contributed to the revision of the final version of the manuscript, and approved the final version submitted.

Funding The study was supported by Indian Council of Medical Research (ICMR), New Delhi, India.

Data Availability Data are available upon reasonable request. For further information please contact madhuerc@gmail.com.

Code Availability NA

\section{Declarations}

Ethics Approval The study protocol, data extraction forms, and procedures were reviewed and approved by the Institutional Ethical Committee of ICMR-National Institute of Virology, Pune, and the ethical committee of Bai Jerbai Wadia Hospital for Children.

Consent to Participate All parents provided written informed consent to participate in the study.

Consent for Publication The informed consent included the authorization to publish information.

Conflict of Interest The authors declare no competing interests.

\section{References}

1. Li X, Xu W, Dozier M, He Y, Kirolos A, Theodoratou E, et al. The role of children in transmission of SARS-CoV-2: a rapid review. J Glob Health. 2020;10(1):011101.

2. Liu P, Cai J, Jia R, Xia S, Wang X, Cao L, et al. Dynamic surveillance of SARS-CoV-2 shedding and neutralizing antibody in children with COVID-19. Emerg Microbes Infect. 2020;9(1):1254-8.

3. Shields AM, Burns SO, Savic S, Richter AG, UK PIN COVID19 Consortium. COVID-19 in patients with primary and secondary immunodeficiency: the United Kingdom experience. J Allergy Clin Immunol. 2021;147(3):870-5. 
4. Aghamohammadi A, Abolhassani H, Kutukculer N, Wassilak SG, Pallansch MA, Kluglein S, et al. Patients with primary immunodeficiencies are a reservoir of poliovirus and a risk to polio eradication. Front Immunol. 2017;8:685.

5. Choi B, Choudhary MC, Regan J, Sparks JA, Padera RF, Qiu X, Solomon IH, Kuo HH, Boucau J, Bowman K, Adhikari UD. Persistence and evolution of SARS-CoV-2 in an immunocompromised host. N Engl J Med. 2020;383(23):2291-3.
Publisher's Note Springer Nature remains neutral with regard to jurisdictional claims in published maps and institutional affiliations. 